\title{
Primary Lesser Omentum Torsion - An Extremely Rare Cause of Acute Abdomen and a Very Uncommon Subtype of Intraperitoneal Focal Fat Infarction (IFFI): Case Report and Review of the Literature
}

\author{
Vasileios K. Mavroeidis $^{\mathrm{a}, \mathrm{b}}$, Dimitrios I. Matthioudakis ${ }^{\mathrm{a}}$, Nikolaos K. Economou ${ }^{\mathrm{a}}$, Ioannis D. Karanikas ${ }^{\mathrm{a}}$
}

\begin{abstract}
We report a case of a 17-year-old patient who presented with sharp epigastric pain and features of acute abdomen and was finally diagnosed and treated during laparotomy where a lesser omentum torsion was detected. Such cases are extremely rare while the clinical manifestations mimic other causes of acute abdomen. On the other side, the technological progress of the imaging techniques has allowed during the last years, the unification of all these entities which present with focal fat tissue necrosis such as torsion and/or infarction of the greater/lesser omentum or an epiploic appendage, under the term 'Intraperitoneal Focal Fat Infarction' (IFFI), due to their common features, encouraging parallelly the conservative treatment due to the spontaneous self-limiting evolution in most cases. The report is followed by a review of the literature regarding the diagnostic means and choices of treatment of these rare conditions.
\end{abstract}

Keywords: Lesser omentum; Omental torsion; Acute abdomen; Epigastric pain; Intraperitoneal Focal Fat Infarction

\section{Introduction}

Torsion of the lesser omentum is an extremely rare situation presenting as acute surgical abdomen with up to 10 cases published up to now with various termins having been used to describe the diagnosis. The first description was made by Von Klimko in 1929 followed by Erdély in 1932 and Black in

Manuscript accepted for publication May 16, 2013

\footnotetext{
${ }^{a} 1$ st Surgical Department, Sismanogleio General Hospital of Attica, 1 Sismanogleiou Str., Marousi, P.C. 15126, Athens, Greece

${ }^{\mathrm{b}}$ Corresponding author: Vasileios Mavroeidis, 1st Surgical Department, Sismanogleio General Hospital of Attica, 1 Sismanogleiou Str., Marousi, P.C. 15126, Athens, Greece. Email: blackbasildr@yahoo.gr
}

doi: http://dx.doi.org/10.4021/jmc1317w
1954 under the title 'torsion of the third omentum' [1]. Bierman and Jones in 1923 recognised a 'Third Omentum' as an anatomical structure and recorded two examples, the first being an accidental finding during laparotomy in a young man with 'chronic appendicitis', and the second, in a man during exploratory laparotomy to find the cause of a pyloric filling defect revealed by X-ray. A veil of lesser omentum hanging in front of the anterior surface of the stomach and attached to the lesser curvature was found in both cases. They assumed that this 'Third Omentum' has a developmental origin due to the persistence into adult life of a fold, resulted by the 'out pouching' of the lesser omentum which is very occasionally seen in the human embryon. This view has been confirmed by several authors although the anatomical abnormality is rather rare [1].

During the last years, the term 'Intraperitoneal Focal Fat Infarction' (IFFI), introduced by van Breda Viersmann in 1999 , has been widely used to describe in a unified fashion all these various conditions such as torsion and/or infarction of the greater/lesser omentum or an epiploic appendage (epiploic appendagitis $[\mathrm{EA}]$ ), who have similar aetiology, clini-

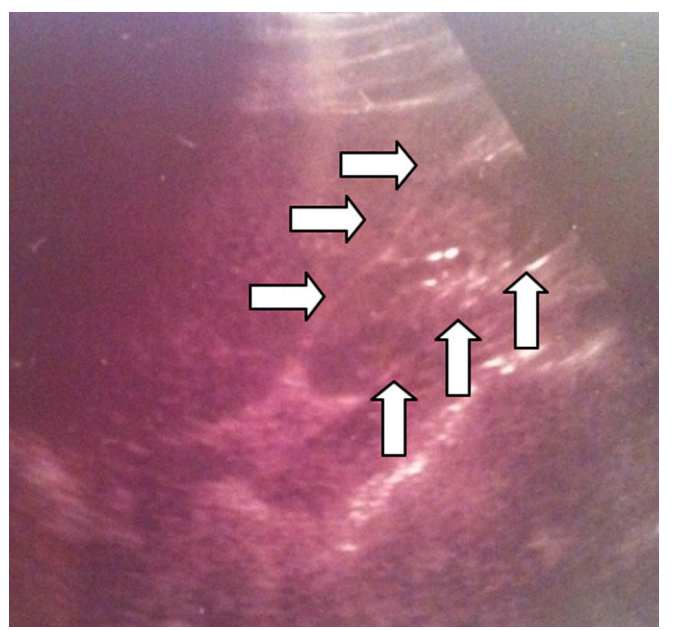

Figure 1. The abdominal U/S was evaluated as uncontributive, however a well circumscribed moderately hyperechoic formation in the anatomic location of the lesser omentum can be noticed (arrows). 

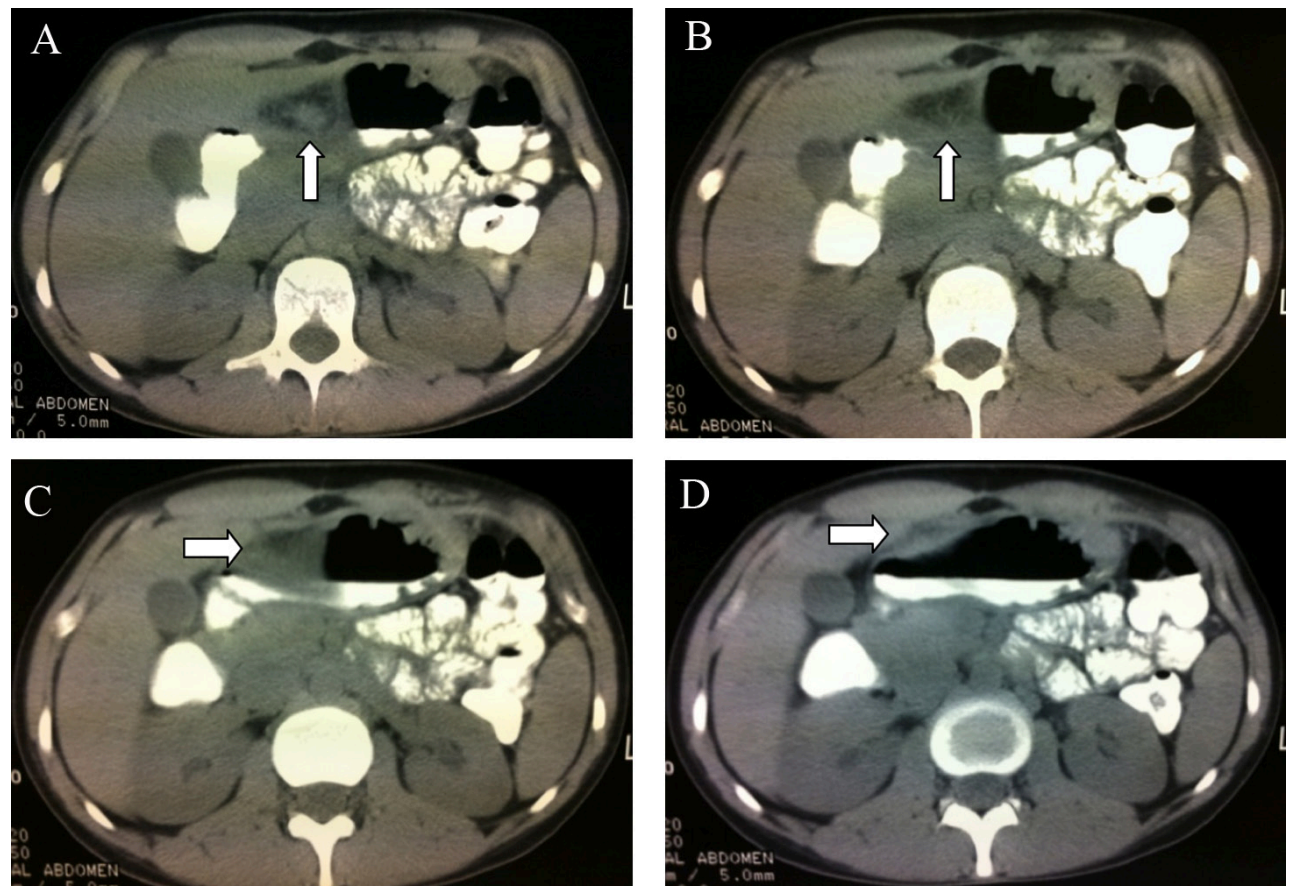

Figure 2. a) A well circumscribed fatty inflammatory mass can be recognised (arrow); b) Hyperechoic streaks within the mass (arrow); c) The formation lying between the posterior edge of the left hepatic lobe and the prepyloric part of the stomach (arrow); d) The formation lying on the gastric antrum (arrow).

cal and radiological features, prognosis and treatment, and only differ by their various anatomical locations and dimensions, while focal fat tissue necrosis is their common point $[2,3]$. Five cases of torsion of the falciform ligament (FL) or a lipomatous appendage of the FL, have also been described [2]. Most reported cases refer to torsion and/or infarction of the greater omentum or epiploic appendages [3]. Nowadays, the diagnosis of IFFI is much more frequent than previously considered, following the continuous progress of U/S and $\mathrm{CT}$ technology with many case reports, small series and pictorial reviews having already been published $[2,3]$. Much interest has been focused lately on the conservative treatment of these situations justified by the spontaneous self-limiting evolution in most cases $[2,3]$.

\section{Case Report}

A 17-year-old Caucasian (Albanian origin) female patient was admitted to the emergency department complaining of acute intense epigastric pain, nausea and anorexia. Her medical history was free. She described the onset of a sharp, continuous pain with increasing intensity 5 hours ago, located in the epigastrium from the start, without irradiation, not related to oral intake of food.

On physical examination her heart rate was $105 / \mathrm{min}$, her temperature was $37.1^{\circ} \mathrm{C}$ and her facet was affected. The abdomen was severely painful on palpation of the epigastrium, with guarding and rebound tenderness.

Laboratory examinations revealed a white blood cell count of $11.50\left(\times 10^{9} / \mathrm{L}\right)$, Neu $68.9 \%$, Ly $20.9 \%$, Mo $9.5 \%$ $\left(1.09 \times 10^{9} / \mathrm{L}\right.$-monocytosis $)$, and a C-reactive protein of 16.1 (IU/L). Urinalysis detected pus cells: 18-20/HPF, Leucocyte esterase: $75 / \mu \mathrm{l}$. All other tests were within normal rates.

The chest and abdominal X-ray did not reveal any pathological findings while the abdominal U/S was evaluated as uncontributive due to intense presence of air in the bowels (Fig. 1).

An abdominal CT described the presence of "a fatty inflammatory mass, containing hyperechoic streaks between the posterior edge of the left hepatic lobe and the prepyloric part of the stomach." Additional findings were linear fluid collection in the right paracolic space and fluid collection in the Douglas space (Fig. 2). The presence of a tamponaded perforation of a peptic ulcer was postulated.

A nasogastric tube was set and initial administration of i.v. fluids was performed. The usual analgetics did not ease the pain and only opiods were effective. Due to the acute and intense continuous features of the condition accompanied with an uncertain diagnosis, an exploratory laparotomy was decided.

A midline incision was performed and a mild serosanguineous fluid collection was initially found and sponged out from the peritoneal cavity. A free lump of the lesser omentum attached with its pedicle to the lesser curvature of the stomach was found rotated at 720 degrees in a clockwise di- 

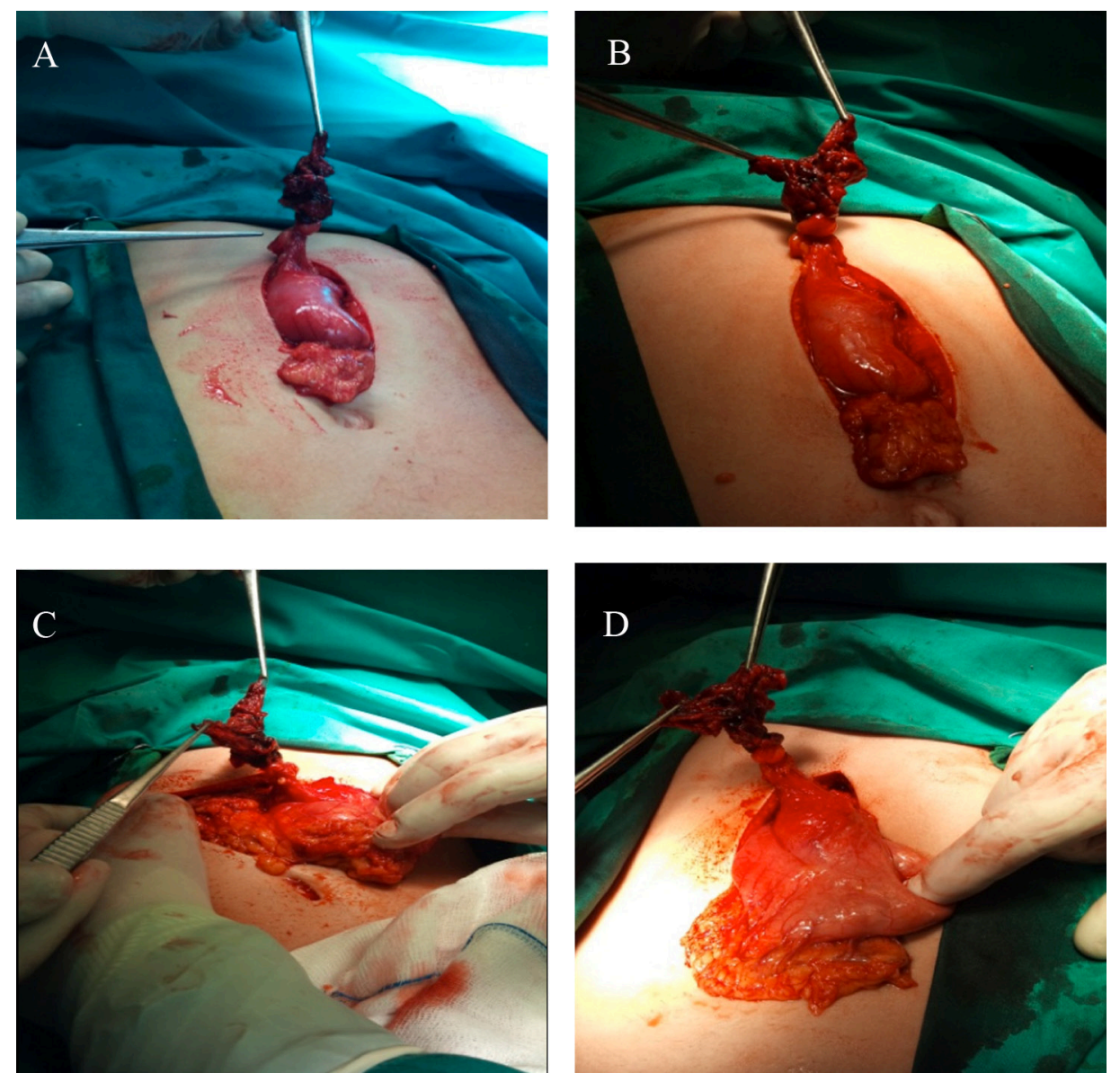

Figure 3. A free part of the lesser omentum and its pedicle originating from the lesser curvature of the stomach is depicted twisted clockwise at $720^{\circ}$, with gangrene of the part lying distally to the level of the torsion. The horizontal axis of the torsion can be clearly seen. The diffuse ischaemic and necrotic parts of the affected lesser omentum are well recognised.

rection, with gangrene of the part lying distally to the level of the torsion, and strongly adhered to the prepyloric part of the stomach. The affected structure was unchained from the stomach and resected from its basis (Fig. 3).

The surgical specimen was sent to biopsy. During laparotomy, an upper GI endoscopy was additionally performed in order to avoid neglect of associated gastric pathology, and the presence of an ulcer was ruled out. However the presence of dilatation of the superficial mucosal vessels (mild gastritis) of the gastric wall underlying to the adhered twisted part of the lesser omentum was noticed. On palpation of the peritoneal cavity no other pathology was noted. The postoperative period was uneventful and the patient was discharged after 4 days in good condition. The histopathological report confirmed the diagnosis and described "haemorrhagic infiltration of the omentum, with congestive and thrombosed vascular branches, inflammatory infiltration, hyperplasia of the mesothelial cells and focal organisation of the inflammation with accompanying fibrosis and hyalinisation of the stroma - findings compatible with omental torsion". Gastroscopy performed one month after surgery did not detect any pathological findings.

\section{Discussion}

Torsion of the lesser omentum is an extremely rare emergency condition that presents as an acute surgical abdomen. Among the situations appearing as IFFI, it remains very infrequent.

Omental torsion and infarction occurs when the organ twists on its vertical axis to such an extent that its vascular perfusion is occlused. Two conditions are prerequisite for torsion to occur: a) A redundant and mobile segment and b) a fixed basis around which the segment can rotate. When torsion happens the severity of histological damage produced, varies from mild vascular constriction leading to oedema, to complete strangulation which implies infarction and frank gangrene [4].

Additionally, omental torsion may be primary or secondary. Primary torsion is characterized by the absence of any associated intra-abdominal pathology. Predisposing fac- 
tors to primary torsion may be anatomical variations such as accessory or bifid omentum, irregular accumulations of omental fat in obese patients, and narrowed omentum pedicle. Redundancy of omental veins may result in kinking around the shorter and tenser arteries. For torsion of the greater omentum, the higher incidence on the right side is explained by the greater size and mobility compared to the left side. On the other side, factors that precipitate omental torsion are those causing displacement of the omentum such as trauma, violent exercise and hyperperistaltism, all increasing the passive movement of the omentum. The precise cause of omental torsion is unknown though [4].

Secondary torsion is associated with pre-existing abdominal pathology and is more frequent. Tumors, cysts, intra-abdominal inflammatory lesions, hernia sacs, postsurgical wounds and scarring, can all be associated with secondary omental torsion [4]. Two cases of secondary torsion and infarction associated with a lipoma of the lesser omentum, have been recently published $[5,6]$.

The initial point in the pathogenesis of the process is the rotation of the omentum around a pivotal point usually in a clockwise direction. The venous circulation is compromised resulting in congestion and oedema of the distal omentum. The implied hemorrhagic extravasation leads to a serosanguineous fluid collection in the peritoneal cavity. As the process progresses, arterial occlusion leads to acute hemorrhagic infarction and resultant necrosis of the omentum. Spontaneous derotation may in some cases occur and this may explain according to some authors the presence of otherwise unjustified adhesions in the right lower quadrant. If no treatment is administered, the natural process of omental torsion is necrosis and fibrosis [4].

A preoperative accurate diagnosis is very rarely set and the previously reported range for greater omentum torsion is $0.6-4.8 \%$ [4]. It is logical that a clinical diagnosis of lesser omentum torsion is much more unlikely preoperatively concerning the extremely low frequency of the disease and the lack of specific signs and symptoms. Adults of either sex are more frequently affected by omental torsion and this statistics seem valid for cases concerning the lesser omentum as well, according to the demographics of the so far reported few cases.

As presented in our case, patients with lesser omentum torsion usually complain of acute sharp pain in the epigastrium and eventually right hypochondrium, with increasing intensity if left untreated, without irradiation, sometimes with a colicky character. Nausea is usually present though vomiting is not often. A low grade fever may be present.

On physical examination the epigastrium is painful with signs of guarding and rebound tenderness.

Laboratory studies usually show mild increase of the inflammatory markers - white blood cell count, C-reactive protein, sedimentation rate.

Abdominal and chest plain radiography do not show specific signs and air in the subdiaphragmatic space is absent.

During the last years great interest has been focused on applying the benefits of the U/S and CT technology progress in evaluating and describing precisely the imaging features of all pathologies presenting with IFFI, in order to offer accurate diagnosis and encourage conservative treatment of these rare entities with common clinical and laboratory characteristics that usually follow a spontaneous benign evolution $[2,3]$. U/S and merely CT have been shown to have a high sensitivity and specificity for the diagnosis of IFFI [2].

Before the advent of cross sectional imaging, segmental infarction of the omentum was extremely rarely diagnosed before surgery. On U/S the classical presentation consists of a solid, incompressible, painful and moderately hyperechoic hyperattenuating mass in the anatomic area of the lesser omentum $[2,3]$.

On CT scans the presentation is also that of a well circumscribed fatty inflammatory mass, surrounded by normal organs, circumscribed or covered by an inflammatory peritoneum and containing hyperechoic streaks probably corresponding to fibrous band and/or dilated thrombosed veins. Integrity of the adjacent organs eliminates the diagnosis of inflammatory fat secondary to other surgical conditions [2, $3]$. The presence of concentric linear streaks at the level of the torsion site is considerated as pathognomonic for an omental torsion but this sign has been extremely rarely described. Thanks to its high capabilities of millimetric MPR, MDCT would probably be able to better demonstrate torsion of epiploic vessels. Because of the good prognosis and spontaneous favourable evolution in most cases, follow-up studies are not systematically performed and in the very rare cases in which follow-up was available, complete resolution on inflammatory changes of both omentum and peritoneum was documented. It is now well-recognized that the diagnosis of IFFI is safer with CT than with US, not only because the signs are more consistent and specific but also because CT, generally definitively excludes other mimicking or associated acute inflammatory abdominal conditions more safely [2].

Retrospectively evaluating the images from the U/S and CT studies of our patient it becomes clear that the criteria for identification as IFFI are fulfilled.

If a gastroscopy is performed in order to exclude the diagnosis of peptic ulcer disease, a mild reactive gastritis of the underlying to the infracted lesser omentum gastric wall might be present as described in our case, but the findings are insufficient to explain the clinical symptomatology.

Differential diagnosis has to be made with peptic ulcer complications, pancreatitis, complicated cholecystolithiasis and appendicitis $[1,2,7]$.

The histopathological presentation of omental infarction depends on the duration of development. Initially, hemorrhagic infarction with fat necrosis is noticed, followed by infiltration by lymphocytes, histiocytes, and finally fibroblasts, 
resulting in fibrosis and scar formation [2].

The optimal treatment has been a matter of controversy. Open surgery, laparoscopic approach and conservative treatment have all been applied with success [1-7]. The advances in U/S and CT technology with exceptional descriptions of all subtypes presenting with IFFI, along with their wide reliability, are a strong argument for those who advocate conservative treatment as it has been confirmed that these similar situations have a favourable evolution when treated only with analgetics and anti-inflammatory drugs $[2,3]$. This type of treatment has gained many supporters during the last decade with confirmed success in non-complicated cases and has been performed in patients with primary lesser omentum torsion as well $[2,3]$.

On the other hand, some authors emphasize on the fact that the extensive use of CT in younger patients and its diagnostic reliability concerning this particular matter may not be justified and therefore suggest that surgical resection remains the treatment of choice in the majority of the cases [7]. Another argument is that the U/S has been shown to be not as sensitive as CT and extremely operator dependant [2], a fact with greater importance when CT is not available. Additionally, after surgery the symptoms resolve rapidly and the possibility of complications such as abscess formation or adhesions is minimized [2]. Either open or laparoscopic approach, have been safely used to set the right diagnosis in cases of uncertainty or uncontributive imaging findings, and apply the definitive treatment by resecting the affected organ, as performed in this case. Postoperative recovery is usually rapid while morbidity is minimal [4].

The progress of imaging techniques along with the increasing use of exploratory laparoscopy for the evaluation of acute abdominal pain may make omental torsion and other cases of IFFI, an even more frequently recognised diagnosis than what has been succeeded until now. Radiologists should become more familiar with the particular imaging characteristics of these rare situations as a significant amount of knowledge has been available on this subject, in order to provide more precise and persuasive diagnoses and essentially help with the choice of treatment. On the other side, surgeons should become more familiar with these rare entities as they can mimic other common diseases presenting with acute sur- gical abdomen, and the possible alternatives of treatment as conservative approach is gaining increasing interest and provides a reasonable choice in most cases.

Torsion of the lesser omentum provides an extremely rare clinical diagnosis but should be included in the differential diagnosis of diseases with epigastric location presenting with acute surgical abdomen. The choice of treatment should be based on the patient's age and clinical condition, the availability of imaging techniques, the radiologist's experience and the surgeon's experience and technical skills.

\section{Conflict of Interest}

The authors declare no conflict of interest.

\section{References}

1. Black AL. Torsion of the third omentum. Postgrad Med J. 1954;30(350):657-658.

2. Coulier B. Contribution of US and CT for diagnosis of intraperitoneal focal fat infarction (IFFI): a pictorial review. JBR-BTR. 2010;93(4):171-185.

3. Coulier B, Van Hoof M. Intraperitoneal fat focal infarction of the lesser omentum: case report. Abdom Imaging. 2004;29(4):498-501.

4. S. Kargar \& R. Fallahnejad: Primary Torsion Of The Lesser Sac Omentum. The Internet Journal of Surgery ISSN: 1528-8242. 2006 Volume 7 Number 2.

5. Pearl JP, Pennypacker JL. Infarction of a lipoma of the lesser omentum: laparoscopic treatment of a rare condition. Surg Laparosc Endosc Percutan Tech. 2011;21(4):e197-199.

6. Hishiki S, Fukushima H, Nishimura T, Horiguchi J, Shibayama T, Chong JM. [A case of lipoma of the lesser omentum with torsion of the pedicle in a twenty-eightyear-old woman]. Nihon Shokakibyo Gakkai Zasshi. 2010;107(9):1450-1455.

7. Bohlman I., De Lorenzi D. Segmental infarction of the lesser omentum and acute abdomen. [Abstract] Chirurgia 2011 August; 24(4):215-217. 